\title{
Gerenciamento de recursos materiais com enfoque na queixa técnica
}

\author{
Management of material resources with a focus on technical complaints
}

Gerenciamiento de recursos materiales con enfoque en la queja técnica

Roseli Broggi Gil ${ }^{1}$, Lucieli Dias Pedreschi Chaves ${ }^{2}$, Ana Maria Laus ${ }^{3}$

\footnotetext{
${ }^{1}$ Enfermeira, Mestre em Enfermagem Fundamental. Enfermeira do Hospital Universitário da Universidade Estadual de Londrina. Londrina, PR, Brasil. E-mail: roseligil@gmail.com.

${ }^{2}$ Enfermeira, Doutora em Enfermagem. Professora Associada da Escola de Enfermagem de Ribeirão Preto da Universidade de São Paulo (EERP/USP). Ribeirão

Preto, SP, Brasil. E-mail: dpchaves@eerp.usp.br.

${ }^{3}$ Enfermeira, Doutora em Enfermagem Fundamental. Professora Associada da EERP/USP. Ribeirão Preto, SP, Brasil. E-mail: analaus@eerp.usp.br.
}

\section{RESUMO}

Estudo descritivo cujo objetivo foi caracterizar o processo de notificação da queixa técnica de material de consumo em hospital de ensino público e integrante da Rede Hospital Sentinela da Agência Nacional de Vigilância Sanitária. Os dados obtidos dos Impressos de Notificações resultaram em 260 notificações analisadas, relativas ao período 2007 a 2009 . Os resultados apontaram predominância de não conformidades no grupo de material médico-hospitalar (80,4\%) e as queixas principais se referiram à estrutura dos produtos utilizados (79\%). Do montante das notificações, 7,7\% foram encaminhados ao Sistema Nacional de Notificações em Vigilância Sanitária. Os enfermeiros foram os profissionais que majoritariamente $(81,2 \%)$ realizaram as notificações. Os achados desta investigação revelaram a relevância da implantação e uso de sistemas de registros sistemáticos de avaliações de materiais, enquanto subsídio para uma gestão eficiente no que concerne à maximização de recursos econômicos.

Descritores: Administração de Materiais no Hospital; Análise Prévia de Produtos; Economia e Organizações de Saúde; Enfermagem; Vigilância Sanitária.

\section{ABSTRACT}

Descriptive study aimed to characterize the process of notification of the technical complaint of consumables in a public teaching hospital, a member of Sentinela Hospital Network of the National Health Surveillance Agency. The data obtained was from the printed notices that resulted in 260 notifications analyzed from 2007 to 2009 . The results showed a predominance of non-conformities in the group of medical-hospital material $(80.4 \%)$ and the main complaints referred to the structure of the products used (79\%). Of the total amount of notifications, $7.7 \%$ were referred to the National System of Notifications in Sanitary Surveillance. The nurses were the professionals who mostly (81.2\%) made the notifications. The findings of this research revealed the importance of implementing and using systems for making systematic records of material evaluations as a basis for efficient management regarding the maximization of economic resources.

Descriptors: Materials Management, Hospital; Previous Analysis of Products; Health Care Economics and Organizations; Health Surveillance.

\section{RESUMEN}

Estudio descriptivo que objetivó caracterizar el proceso de notificación de queja técnica de material de consumo en hospital de enseñanza pública, miembro de la Red Hospitalaria Centinela de la Agencia Nacional de Vigilancia Sanitaria. Los datos obtenidos de los Impresos de Notificaciones totalizaron 260 notificaciones analizadas, relativas al período 2007 a 2009. Los resultados expresaron predominancia de inconformidad en el grupo de material médico-hospitalario (80,4\%) y las quejas principales se refirieron a la estructura de los productos utilizados $(79 \%)$. Del total de notificaciones, el $7,7 \%$ fue derivado al Sistema Nacional de Notificaciones en Vigilancia Sanitaria. Los enfermeros fueron quienes realizaron mayoritariamente las notificaciones $(81,2 \%)$. Los hallazgos de esta investigación revelaron la importancia de implantar y utilizar sistemas de registro informático de evaluaciones de materiales como respaldo de una gestión eficiente en lo referente a la optimización de recursos económicos.

Descriptores: Administración de Materiales de Hospital; Análisis Previo de Productos; Economía en Atención de Salud y Organizaciones; Vigilancia Sanitaria. 


\section{INTRODUÇÃo}

O atual panorama nacional mostra um contínuo crescimento com o gasto na saúde, não relacionado somente à universalização do acesso, ocorrido a partir da criação do Sistema Único de Saúde (SUS) e à incorporação tecnológica das últimas décadas, mas também decorrentes das questões gerenciais ${ }^{(1)}$. Neste sentido, os gestores de serviços públicos têm vivenciado dificuldades para administrarem as instituições com os escassos recursos financeiros repassados pelo governo, em virtude "[...] da diminuição dos gastos federais com saúde, frente às demandas da população por serviços de saúde."(2).

Os hospitais, do ponto de vista organizacional, possuem características diferentes de qualquer outra organização. Entre elas destaca-se a sua complexidade, de funcionarem ininterruptamente e por interagirem com pessoas em momentos de fragilidade, quando necessitam de assistência específica e, em muitos casos, de recursos sofisticados. A incorporação de novas tecnologias remete à responsabilidade de ofertar maior segurança para o paciente e profissional prestador do cuidado, porém os processos de trabalho também precisam ser revistos, com treinamento contínuo, tanto nos relacionados ao cuidado direto como nos sistemas de gerenciamento de recursos materiais.

A somatória dos gastos com recursos humanos e materiais contribui para a elevação dos custos hospitalares $^{(3)}$, fato esse vivenciado tanto nas instituições públicas como nas privadas. O valor da somatória dos custos diretos e indiretos varia de "[...] 35\% a 45\% do orçamento total do hospital, e aumentam em decorrência do grau de desorganização do sistema de materiais"(4). Os recursos materiais são responsáveis por uma parcela significativa dos gastos nas instituições de saúde, sendo que os custos diretos de aquisição "[...] situam-se entre $23 \%$ e $29 \%$, dependendo da complexidade e da organização da instituição"(4). Esses dados dimensionam a relevância da adoção de ferramentas voltadas para o gerenciamento desses recursos, tanto do ponto de vista econômico quanto dos resultados advindos da sua utilização, além de evidenciar que a busca pelo conhecimento sobre custo nessa área é fundamental para a sobrevivência das instituições prestadoras de assistência à saúde. Sendo assim, temas como eficiência e eficácia das atividades devem ser trabalhados em conjunto com aspectos de qualidade e segurança, bem como o uso racional dos recursos, evitando-se o desperdício ${ }^{(5)}$. Em momento algum as questões financeiras e culturais devem sobrepor os aspectos assistenciais de qualidade e de segurança.
No Brasil, "[...] o controle sanitário dos produtos, incluindo todas as etapas de seu ciclo vital, da fabricação à sua comercialização, "[...] são de competência da Agência Nacional de Vigilância Sanitária (ANVISA)"(6). Ela se responsabiliza pela regulamentação e regulação dos produtos como forma de promover a vigilância em saúde e subsidia as instituições no que se refere ao controle dos produtos comercializados. Portanto, a comercialização de produtos para uso em saúde está vinculada às legislações específicas e estes agrupados em cinco categorias: produtos para diagnósticos de uso in vitro; produtos para saúde (materiais e equipamentos), saneantes domissanitários, produtos de higiene pessoal, cosméticos e perfumes, e medicamentos.

A ANVISA ${ }^{(7)}$ define produtos para saúde como "[...] produtos utilizados na realização de procedimentos médicos, odontológicos e fisioterápicos, bem como no diagnóstico, tratamento, reabilitação ou monitoração de pacientes".

Decorrente do aumento da incorporação em grande escala de diferentes tecnologias no processo de trabalho de assistência à saúde surgiu a necessidade de implantação de sistemas de monitoramento que possibilitassem a identificação, de forma precoce, de problemas técnicos - Desvio de Qualidade/Queixa Técnica (QT), de modo a minimizar a ocorrência de eventos adversos.

No contexto da Vigilância Sanitária ${ }^{(8)}$, "Evento Adverso é considerado qualquer efeito não desejado, em humanos, decorrente do uso de produtos sob vigilância sanitária", e a "Queixa Técnica, entendida como qualquer notificação de suspeita de alteração ou irregularidade de um produto ou empresa, relacionada a aspectos técnicos ou legais, que poderá ou não causar dano à saúde individual ou coletiva".

O relato da ocorrência de QT e EA não se limita apenas ao nosso país e nos tempos atuais. Os países desenvolvidos, como os Estados Unidos da América do Norte e Membros da Comunidade Europeia, já adotam medidas mais severas desde a década de $1980^{(6)}$.

A literatura nacional tem apontado sobre a comercialização de material de consumo hospitalar com qualidade insatisfatória e questionável, exigindo dos órgãos competentes a adoção de algumas medidas fiscalizatórias ${ }^{(9-11)}$. Uma forma de se evitar esta situação é a implantação do processo de pré-qualificação, enquanto estratégia para verificação de conformidade do produto antes de sua aquisição. Trata-se de uma ferramenta para o gerenciamento de risco, uma vez que passa a atuar como uma barreira contra a entrada de produtos com 
qualidade duvidosa, porém deve ser sistematizada e $\operatorname{acurada}^{(10,12)}$.

A legislação vigente, entretanto, não garante, em sua totalidade, que a segurança seja mantida após a obtenção do registro ${ }^{(6)}$. Neste cenário, medidas de controle na etapa de pós-comercialização dos produtos de uso na saúde passam a ter grande relevância. A vigilância póscomercialização (Vigipos) de produtos para a saúde, é definida como "Sistema de Notificação e Investigação em Vigilância Sanitária para o monitoramento, análise e investigação dos eventos adversos e queixas técnicas relacionadas aos serviços e produtos sob vigilância na fase de pós-comercialização/pós-uso"(8).

Assim, a notificação da queixa técnica tem se constituído em ferramenta importante na gestão, subsidiando a ANVISA na adoção de medidas, uma vez que permite identificar irregularidades de produto e/ou fabricante no tocante a desvio de qualidade e orienta as instituições de saúde para os processos de análise e de vigilância das novas tecnologias e recursos disponíveis. Entretanto, tem sido um desafio a obtenção de informações confiáveis que possibilitem o monitoramento sistemático da qualidade do material de consumo hospitalar na fase de pós-comercialização. Nesta perspectiva, esta investigação foi desenvolvida com o objetivo de caracterizar o processo de notificação da queixa técnica de material de consumo hospitalar, no contexto do gerenciamento de recursos materiais de um hospital público de grande porte.

\section{METODOLOGIA}

Trata-se de estudo descritivo, retrospectivo, de abordagem quantitativa, com coleta de dados realizada pela Assessoria de Enfermagem no Controle de Recursos Materiais (AECRM) da Diretoria de Enfermagem de um Hospital de ensino do Estado do Paraná. Esta instituição presta atendimento ambulatorial e hospitalar de alta complexidade, possui 316 leitos - todos conveniados ao SUS - e integra a Rede Brasileira de Hospitais Sentinela da ANVISA desde 2002.

Antecedendo a coleta de dados, o estudo foi autorizado pela instituição hospitalar e posteriormente aprovado pelo Comitê de Bioética e Ética em Pesquisa do Hospital investigado (CAAE no 0197.0.268.153-09).

Os dados foram obtidos pelo pesquisador principal deste estudo, que atua na AECRM, e extraídos dos Impressos de Notificação instituídos pela Gerência de Risco para formalização das queixas técnicas e/ou eventos adversos dos materiais de consumo hospitalar, referentes ao período de janeiro de 2007 a dezembro de 2009. Para fins de inclusão na pesquisa, definiu-se como critérios o registro da queixa estar documentado no impresso específico e estar disponível no arquivo documental da unidade.

As variáveis de interesse nesta investigação referiram-se ao tipo de produto; grupo de material no qual estava inserido; tipo de ocorrência relatada; categoria do problema; mês e ano da ocorrência; setor que registrou o problema; categoria profissional do notificador; e notificações informadas ao sistema NOTIVISA.

O material de consumo hospitalar refere-se a todo produto utilizado para prestar assistência direta ao paciente em atendimento ou internado, de uso único, padronizado e adquirido na instituição seguindo a legislação vigente. E foram categorizados nesta pesquisa em três grupos: material médico-hospitalar, material para higiene pessoal e para uso no processo de esterilização.

A variável "categoria do problema" foi analisada quanto a embalagem, estrutura e/ou aspecto alterado, baseada nos critérios adotados pela ANVISA ${ }^{(8)}$.

Em relação à embalagem foram consideradas como não conformidade problemas na identificação, quantidade de unidades diferente do informado na embalagem, falha na selagem, tamanho inadequado para o produto e/ou para abertura do material sem infringir a técnica asséptica. Quanto à estrutura, presença de rachadura, quebra do produto ou parte dele, encaixe, obstrução, vazamento, tamanho, absorção, perda de corte e a presença de corpo estranho. Para o item aspecto alterado, cor, odor e mancha. Diante da possibilidade de simultaneidade de categorias, foram adotadas ainda duas outras modalidades de enquadramento, embalagem e estrutura, e aspecto alterado e estrutura. Quando da impossibilidade de enquadramento nas categorias estabelecidas, definiu-se por uma denominada não se aplica.

Os dados, lançados em um instrumento de coleta específico para esta investigação, foram codificados para fins de transcrição para o Epi Info, versão 3.3.2, e, analisados, utilizando-se estatística descritiva.

\section{RESULTADOS}

No período investigado foram recebidas 409 notificações de queixa técnica, sendo que 260 atenderam aos critérios de inclusão. Dentre as 149 excluídas, 72 $(48,32 \%)$ não foram localizadas no arquivo documental.

Quanto ao contingente de materiais de consumo hospitalar notificados, verificou-se, nos três grupos criados, que o material médico-hospitalar abrangia 33 itens informados, correspondendo a $80,4 \%$ das queixas; 
material para higiene pessoal, cinco itens, com 15,8\% e material para uso no processo de esterilização, composto apenas por um tipo de material, representando $3,8 \%$.
$\mathrm{Na}$ Tabela 1 destacam-se apenas os materiais notificados com percentuais mais expressivos, seguindo a classificação por grupo de material e ano.

Tabela 1: Distribuição dos produtos mais notificados, de acordo com o grupo de material e ano. Paraná, Brasil, $2007 / 2009$.

\begin{tabular}{|c|c|c|c|c|c|c|c|c|}
\hline \multirow{2}{*}{$\begin{array}{c}\text { Grupo de Material } \\
\text { Material Médico-Hospitalar }\end{array}$} & \multicolumn{2}{|c|}{2007} & \multicolumn{2}{|c|}{2008} & \multicolumn{2}{|c|}{2009} & \multicolumn{2}{|c|}{ Total } \\
\hline & $\mathbf{N}$ & $\%$ & $\mathbf{N}$ & $\%$ & $\mathbf{N}$ & $\%$ & $\mathbf{N}$ & $\%$ \\
\hline 1. Conector três vias & 6 & 13,05 & 6 & 8,33 & 0 & - & 12 & 5,74 \\
\hline 2. Dispositivo para acesso venoso periférico e central & 6 & 13,05 & 6 & 8,33 & 12 & 13,19 & 24 & 11,48 \\
\hline 3. Equipo & 4 & 8,70 & 5 & 6,94 & 10 & 10,99 & 19 & 9,09 \\
\hline 4. Fio de sutura & 3 & 6,53 & 4 & 5,55 & 5 & 5,49 & 12 & 5,74 \\
\hline 5. Luva cirúrgica & 1 & 2,17 & 18 & 25,00 & 18 & 19,78 & 37 & 17,70 \\
\hline 6. Luva procedimento estéril e não estéril & 2 & 4,35 & 3 & 4,17 & 5 & 5,49 & 10 & 4,78 \\
\hline 7. Seringa & 6 & 13,05 & 5 & 6,94 & 6 & 6,59 & 17 & 8,13 \\
\hline 8. Sonda aspiração, enteral, foley e uretral & 6 & 13,05 & 4 & 5,55 & 5 & 5,49 & 15 & 7,18 \\
\hline 9. Demais produtos & 12 & 26,05 & 21 & 29,19 & 30 & 32,98 & 63 & 30,16 \\
\hline Subtotal & 46 & 100,00 & 72 & 100,00 & 91 & 100,00 & 209 & 100,00 \\
\hline Material Para Higiene Pessoal & $\mathbf{N}$ & $\%$ & $\mathbf{N}$ & $\%$ & $\mathbf{N}$ & $\%$ & $\mathbf{N}$ & $\%$ \\
\hline 1. Fralda descartável & 1 & 14,29 & 3 & 33,33 & 6 & 24,00 & 10 & 24,39 \\
\hline 2. Papel toalha interfolha & 4 & 57,13 & 6 & 66,67 & 18 & 72,00 & 28 & 68,29 \\
\hline 3. Demais produtos & 2 & 28,58 & 0 & - & 1 & 4,00 & 3 & 7,32 \\
\hline Subtotal & 7 & 100,00 & 9 & 100,00 & 25 & 100,00 & 41 & 100,00 \\
\hline Material Para Uso no Processo De Esterilização & $\mathbf{N}$ & $\%$ & $\mathbf{N}$ & $\%$ & $\mathbf{N}$ & $\%$ & $\mathbf{N}$ & $\%$ \\
\hline $\begin{array}{l}\text { 1. Embalagem para acondicionamento de material para } \\
\text { ser esterilizado }\end{array}$ & 0 & - & 3 & 100,00 & 7 & 100,00 & 10 & 100,00 \\
\hline Subtotal & $\mathbf{0}$ & - & 3 & 100,00 & 7 & 100,00 & 10 & 100,00 \\
\hline Total & 53 & 100,00 & 84 & 100,00 & 123 & 100,00 & 260 & 100,00 \\
\hline
\end{tabular}

O percentual total obtido, para cada um dos demais produtos médico-hospitalares notificados está apresentado a seguir em ordem decrescente: coletor para diurese sistema fechado e atadura de crepe $(4,31 \%)$; agulha $(2,87 \%)$; sistema de drenagem torácica $(1,91 \%)$; bolsa para coleta de sangue, cânula endotraqueal, compressa de gaze, extensor para equipo e filtro bacteriológico (1,44\%); campo cirúrgico impermeável, cera para osso, linha arteriovenosa e pasta adesiva para exame (0,96\%); avental descartável, cânula traqueostomia, cateter epidural, circuito de pressão positiva contínua nasal - CPAP, compressa de campo operatório, drenos (penrose e Kher), dispositivo urinário, haste flexível para coleta de exame, lâmina de bisturi, máscara modelo bico de pato, sistema fechado para aspiração e tubo de látex $(0,48 \%)$. Para o grupo de material para higiene pessoal obteve-se 2,44\% para aparelho de barbear, papel higiênico e álcool gel antisséptico.

Os aspectos relativos à estrutura do material obtiveram os maiores percentuais de não conformidade/desvio de qualidade, $(76,15 \%)$, segundo categoria da queixa técnica.

No tocante ao produto, constataram-se problemas relativos à sua estrutura nos três grupos de material, porém com destaque acentuado para o material médicohospitalar (78,95\%), conforme apresentado na Tabela 2.

Tabela 2: Distribuição da categoria do problema analisado e relacionado com o grupo de material. Paraná, Brasil, 2007/2009.

\begin{tabular}{|c|c|c|c|c|c|c|c|c|}
\hline \multirow{3}{*}{$\begin{array}{r}\text { Categoria do } \\
\text { Problema } \\
\text { Grupo de } \\
\text { Material }\end{array}$} & \multicolumn{8}{|c|}{ Categoria do Problema } \\
\hline & \multicolumn{2}{|c|}{$\begin{array}{l}\text { Material Médico- } \\
\text { Hospitalar }\end{array}$} & \multicolumn{2}{|c|}{$\begin{array}{c}\text { Material para } \\
\text { Higiene Pessoal }\end{array}$} & \multicolumn{2}{|c|}{$\begin{array}{c}\text { Material para Uso no Processo } \\
\text { de Esterilização }\end{array}$} & \multicolumn{2}{|c|}{ Total } \\
\hline & $\mathbf{N}$ & $\%$ & $\mathbf{N}$ & $\%$ & $\mathbf{N}$ & $\%$ & $\mathbf{N}$ & $\%$ \\
\hline Embalagem & 20 & 9,57 & - & - & - & - & 20 & 7,69 \\
\hline Estrutura & 165 & 78,95 & 24 & 58,54 & 9 & 90 & 198 & 76,15 \\
\hline Aspecto alterado & 9 & 4,3 & 3 & 7,32 & - & - & 12 & 4,62 \\
\hline $\begin{array}{l}\text { Embalagem e } \\
\text { estrutura }\end{array}$ & 10 & 4,79 & 1 & 2,44 & - & - & 11 & 4,23 \\
\hline $\begin{array}{l}\text { Estrutura e aspecto } \\
\text { alterado }\end{array}$ & 1 & 0,48 & 13 & 31,7 & - & - & 14 & 5,39 \\
\hline Não se aplica & 4 & 1,91 & - & - & 1 & 10 & 5 & 1,92 \\
\hline Total & 209 & 100 & 41 & 100 & 10 & 100 & 260 & 100 \\
\hline
\end{tabular}


A Divisão de Centro Cirúrgico, a Unidade de Tratamento Intensivo Neonatal representando um setor da Divisão Materno Infantil, e a Divisão de Atendimento, compreendendo as unidades de Endoscopia, Hemodiálise, Hemodinâmica e Radiologia, foram os locais da instituição que mais notificações encaminharam.

Outro dado relevante refere-se à identificação dos enfermeiros enquanto a categoria profissional que, majoritariamente $(81,2 \%)$, elaborou e encaminhou as informações relativas a não conformidades de material médico-hospitalar.

Constatou-se ainda que $7,7 \%$ das queixas técnicas recebidas, por se enquadrarem na área de tecnovigilância foram encaminhadas ao Sistema Nacional de Notificações em Vigilância Sanitária (NOTIVISA).

\section{DISCUSSÃO}

O gerenciamento de recursos materiais na saúde constitui-se em tema de importância crescente, em decorrência não só dos avanços tecnológicos e de insumos na indústria farmacêutica e de materiais e equipamentos, mas de questões relacionadas ao processo administrativo das organizações, à ausência de sistema de controle, de consumo, desperdício e custo, além de aspectos fundamentais do cuidado, como qualidade e segurança ${ }^{(13)}$.

Diante do significativo percentual das despesas de custeio das instituições de saúde, evidencia-se a magnitude e complexidade do seu gerenciamento, que compreende os processos de programação (padronização, descritivo técnico, previsão), aquisição, armazenamento, distribuição e controle, uso e monitoramento.

O desafio está em equilibrar receita e despesa, e para tal se impõe a implantação de sistemas operacionais na estrutura administrativa, que possibilitem o gerenciamento pautado por resultados eficientes. Dentre estes, pode-se considerar o processo de notificação da queixa técnica de material de consumo de uso hospitalar, enquanto uma ferramenta auxiliar da gestão de materiais, na perspectiva de que produz informações como subsídios para a tomada de decisão.

O Impresso de Notificação instituído pode ser comparado aos documentos que compõem o prontuário de um paciente, frente ao seu valor e importância, para sistematizar as ações e condutas pertinentes às ocorrências com os produtos. Possibilita a investigação dos fatos relatados e seus desdobramentos, inclusive a notificação por meio do sistema Notivisa; subsidia o parecer técnico do produto durante o processo licitatório; permite a construção de banco de dados, de análises estatísticas, entre outras ${ }^{(14)}$.
O número de queixas técnicas obtidas pela instituição hospitalar pesquisada, a partir da adoção desta sistemática, pode ser considerado um indicador de qualidade institucional. As informações fornecidas são importantes e significativas para subsidiar as investigações internas, inclusive para o rastreamento do produto. Entretanto, o número expressivo de IN não localizados quando da coleta de dados, evidencia uma lacuna importante referente ao sistema de documentação. Isso sinaliza para a necessidade de uma reavaliação dessa etapa do processo de trabalho. O hospital optou pela disponibilização do referido impresso na intranet, com projeto de implantação na Web em curto prazo, facilitando ao notificador acompanhar a tramitação do seu relato, na expectativa de que possa estimular o envio de novas notificações, quando da ocorrência/observação de irregularidades com os produtos. Esta medida também tem por objetivo agilizar as condutas por parte da AECRM e da Gerência de Risco Hospitalar.

Um aspecto a ser ressaltado é que por se tratar de um hospital de ensino, torna-se imprescindível manter o bom funcionamento do fluxo dos materiais de consumo para que a falta destes não comprometa, além dos processos assistenciais, os processos de ensinoaprendizagem $^{(3)}$. Mas para tal, torna-se fundamental a participação dos profissionais para a garantia da qualidade e da segurança da atenção à saúde. Para as instituições que fazem parte do Projeto Hospital Sentinela, compete à Gerência de Risco, de acordo com cada caso, adotar as providências necessárias, ou seja, "[...] controlando ou eliminando o risco de exposição de pacientes e trabalhadores a esses produtos, enquanto as investigações mais aprofundadas são levadas à frente pelos órgãos competentes" ${ }^{\prime 15)}$. Os hospitais que ainda não tenham esse serviço instituído podem e devem informar as queixas técnicas ou eventos adversos à ANVISA pelos meios de comunicação (telefone/e-mail/site).

Alguns autores ${ }^{(16)}$ afirmam que $o$ sucesso no gerenciamento de materiais depende em grande parte do envolvimento dos profissionais diretamente ligados às áreas onde ocorre o efetivo consumo, particularmente nos aspectos relativos ao controle e utilização adequada, de forma a reduzir desperdícios e melhorar a eficiência.

Independentemente do curso, as instituições de ensino da área da saúde desempenham importante papel na formação quanto a sensibilização dos diferentes profissionais no que se refere a racionalização no uso dos recursos materiais para as diferentes práticas em serviço, o que sugere a inclusão nos seus currículos de temas relacionados ao gerenciamento de recursos materiais ${ }^{(17)} \mathrm{e}$ 
custos, abordando, de forma contundente, a conscientização e a responsabilidade ética e legal que cada um representa no processo do gerenciamento de risco na atenção à saúde. Desta forma, acredita-se que os novos profissionais sejam os multiplicadores para a conscientização dos gestores e fomentar a implantação de serviços de forma estruturada, visando à aquisição de produtos com qualidade, que propiciem segurança e com preços justos de mercado.

O sucesso de qualquer sistema depende fundamentalmente do comprometimento dos profissionais, tanto para o fornecimento quanto para a utilização das informações. Elas subsidiarão muitas decisões visando à melhoria contínua da qualidade, quando da aquisição de produtos. Para tanto, é necessária a participação de todos durante a avaliação criteriosa, principalmente do material sujeito à vigilância sanitária, com uma visão voltada para a segurança do paciente e dos prestadores da assistência.

Apesar do estímulo para formalização da queixa técnica na instituição investigada, é possível inferir que haja subnotificação, visto que muitos produtos de uso comum às áreas assistenciais não terem sido formalizada a sua notificação por parte de vários setores. Infelizmente, a subnotificação é fato na realidade brasileira ${ }^{(6)}$. Alguns autores atribuem como causas a "[...] escassez e sobrecarga do pessoal nos estabelecimentos de saúde e de tempo para notificar os problemas identificados"(9).

As unidades assistenciais classificadas como críticas (Centro Cirúrgico e UTI Neonatal) foram as que se destacaram no número de notificações enviadas. Tradicionalmente são setores que detêm o uso de recursos materiais mais sofisticados, ou seja, a tecnologia de ponta é inerente ao processo de trabalho e é considerada vital para o desenvolvimento das atividades. Tendo em vista esta característica, bem como o volume de materiais utilizados nos diferentes processos assistenciais, é possível inferir que estas áreas tenham desenvolvido maiores habilidades de observação e avaliação desses itens. O processo de cuidar nas unidades especializadas depende em grande parte do uso racional e seguro de materiais e equipamentos de suporte à vida, entre outros procedimentos, que também requerem políticas eficientes de manutenção efetiva ${ }^{(18)}$.

Nesta investigação, os resultados apontaram, com destaque, os itens do grupo de material médicohospitalar, representados por produtos básicos (luvas, dispositivos para acesso venoso, equipos, seringas, entre outros) entre os materiais que apresentam não conformidade, evidenciando que esse segmento ainda merece maior atenção do ponto de vista da vigilância sanitária, principalmente na fase da pós-comercialização. Certamente há necessidade de se considerar que esse grupo compõe o conjunto de materiais cujos artigos representam alto risco para os usuários em virtude da sua utilização em larga escala.

As queixas técnicas relativas às luvas cirúrgicas e de procedimento coincidem com relatos de outras instituições hospitalares do país. Esses fatos subsidiaram para que a ANVISA publicasse a Resolução da Diretoria Colegiada RDC 5/2008 ${ }^{(19)}$, que estabelece os requisitos mínimos de identidade e qualidade para os tipos de luvas sob regime de vigilância sanitária.

As notificações em relação aos equipos também foram detectadas pela ANVISA, por meio do Sistema Notivisa, sendo o produto com maior número de notificações na questão de sua estrutura, destacando-se falhas de gotejamento e vazamento( ${ }^{(9)}$. Estudo multicêntrico foi desenvolvido em diversos hospitais brasileiros $e$ coordenado pela Unidade de Tecnovigilância/Nuvig/Anvisa, com o objetivo de préqualificar os equipos ${ }^{(10)}$. Os resultados permitiram à ANVISA publicar a RDC 04/2011(20) que estabelece os requisitos mínimos de identidade e qualidade para os equipos de uso único de transfusão, de infusão gravitacional e de infusão para uso com bomba de infusão. Em relação às seringas hipodérmicas, também foi publicada a RDC 03/2011 ${ }^{(21)}$ visando melhorar a qualidade no processo industrial desses produtos e, consequentemente, garantir segurança ao paciente.

Outro achado desta investigação refere-se a identificação do profissional notificador, com destaque para os enfermeiros, evidenciando o envolvimento dessa categoria profissional na avaliação de recursos materiais nesta instituição. A literatura refere a participação marcante da enfermagem no processo de gerenciamento de materiais, a partir da constatação de que essa equipe é a maior requisitante e usuária dos produtos, principalmente dos itens classificados como médicohospitalar ${ }^{(22)}$.

Na dimensão gerencial do processo de trabalho do enfermeiro, as questões pertinentes aos recursos materiais e equipamentos expressam a preocupação com a qualificação da assistência de enfermagem, pois configuram a finalidade do cuidado de enfermagem, além do gerenciamento de custos para a instituição ${ }^{(17,23)}$. Dessa forma, acredita-se que, pela vivência assistencial do enfermeiro, ele seja ator principal na gestão do material de consumo hospitalar, garantindo a eficiência e eficácia da assistência de enfermagem com a utilização de 
produtos que propiciem segurança para o paciente e para o prestador do cuidado, além de articular-se de forma mais objetiva com as áreas administrativas e de apoio do hospital ${ }^{(24)}$.

A natureza do trabalho na assistência aos pacientes propicia o desenvolvimento de sensibilidade crítica por parte dos profissionais. Isto permite que eles se tornem mais atentos aos detalhes pertinentes à qualidade dos produtos, na perspectiva de oferecer aos pacientes os melhores materiais pelo menor custo.

Os avanços e a incorporação de novas tecnologias caracterizam a importância e responsabilidade das instituições perante a Rede Brasileira de Hospitais Sentinela - ANVISA, no contexto do monitoramento das queixas técnicas / eventos adversos dos produtos sob vigilância sanitária (tecnovigilância, farmacovigilância, hemovigilância, saneantes e cosméticos) na fase de póscomercialização ${ }^{(25)}$.

A vigilância aos quesitos necessários à aquisição do material de consumo hospitalar implica não apenas o desperdício de recursos, mas constitui-se em infração sanitária que envolve aspecto da legislação e do Código Penal ${ }^{(10)}$.

Um dos objetivos do monitoramento da póscomercialização é fornecer informações para a ANVISA como forma de promover a vigilância em saúde e subsidiar as instituições no que se refere ao controle de produtos comercializados. Desta forma, o Impresso de Notificação possibilita uma avaliação/feedback do desempenho dos diferentes materiais na fase de pós-aquisição, subsidiando a investigação e a formalização da queixa técnica e/ou evento adverso para o Notivisa, também respaldando a área de parecer técnico quanto à desclassificação de proposta de licitante em aquisições futuras. Além disso, este instrumento fortalece as atividades da gestão de material, pois aproxima o usuário do responsável pelo parecer técnico, fortalecendo o comprometimento com questões pertinentes ao gerenciamento de risco.

\section{CONCLUSÃO}

A tônica no setor saúde está sendo ditada pela restrição de recursos frente a uma demanda cada dia maior, mais abrangente e exigente. $O$ aumento dos gastos na saúde tem relação direta com o aumento dos custos dos serviços prestados. Diminuir custos em hospitais é uma tarefa delicada pela própria natureza dos serviços prestados. Apesar dessas dificuldades, o gerenciamento de custos hospitalares, com vistas à otimização dos recursos, é necessário e um importante fator para o tão almejado equilíbrio financeiro e o aumento da qualidade da atenção prestada.

Em função dessa complexidade, os hospitais necessitam de administração qualificada e preparada para enfrentar os problemas relativos ao gerenciamento de seus recursos.

O processo de notificação orienta para uma análise das causas de eventos adversos e das queixas técnicas, possibilitando investigação de não conformidade, presente nos materiais e necessária para a área da tecnovigilância. Isto deve ocorrer na direção de sempre oferecer ao paciente e profissional prestador de cuidado produtos que apresentem as especificidades/requisitos necessários para cada procedimento.

Os achados desta investigação revelaram a relevância da implantação e uso de sistemas de registros sistemáticos de avaliações de materiais, enquanto subsídio para uma gestão eficiente no que se refere à maximização de recursos econômicos.

O monitoramento dos produtos na etapa de póscomercialização, por meio do IN, deve ser contínuo e de maneira a identificar precocemente as necessidades de intervenção, bem como a sistematização das ações e condutas adotadas.

Além disso, possibilita a implementação de um sistema de rastreabilidade para todos os materiais, permitindo a adoção de medidas precoces que minimizem o impacto de falta dos recursos materiais na instituição decorrente de uma programação mal dimensionada dos itens, perda do controle de estoque, desconhecimento total do consumo pela ausência de dados e controles confiáveis e desperdício de material pela má qualidade, inclusive levando a um desgaste intenso por parte dos profissionais envolvidos. O gerenciamento de risco deve estar no cotidiano dos profissionais da área da saúde, pois se torna uma responsabilidade de todos e não somente dos gerentes, e repercute de forma direta no processo assistencial, devendo ser executado segundo princípios éticos e morais.

\section{AGRADECIMENTO E FINANCIAMENTO}

Trabalho extraído da dissertação "O processo de notificação da queixa técnica de material de consumo de uso hospitalar no contexto do gerenciamento de recursos materiais em um hospital universitário público", do Programa de Pós-Graduação em Enfermagem Fundamental da Escola de Enfermagem de Ribeirão Preto da Universidade de São Paulo, 2011. Bolsista do Conselho Nacional de Desenvolvimento Científico e Tecnológico (CNPQ) - 2009-2011. 


\section{REFERÊNCIAS}

1. Vecina Neto G, Reinhardt Filho W. Gestão de recursos materiais e de medicamentos. São Paulo: FSP/USP; 1998. 2. Castilho V, Castro LC, Couto AT, Maia FOM, Sasaki NY, Nomura $\mathrm{FH}$ et al. Levantamento das principais fontes de desperdício de unidades assistenciais de um hospital universitário. Rev. esc. enferm. USP [Internet]. 2011 [cited 2014 Jul 27]; 45(spe):1613-1620. Available from: http://dx.doi.org/10.1590/S0080-62342011000700012. 3. Paschoal MLH, Castilho V. Implementação do sistema de gestão de materiais informatizado do Hospital Universitário da Universidade de São Paulo. Rev. esc. enferm. USP [Internet]. 2010 [cited 2013 Nov 04]; 44( 4 ): 984-988. Available from: http://dx.doi.org/10.1590/S0080-62342010000400018. 4. Reinhardt Filho W. Gestão de suprimentos e medicamentos. In: Vecina Neto G, MALIK AM, organizadores. Gestão em Saúde. Rio de Janeiro: Guanabara Koogan; 2011. p.191-202. 5. Lopes LA, Dyniewicz AM, Kalinowski LC. Gerenciamento de materiais e custos hospitalares em UTI neonatal. Cogitare Enfermagem [Internet]. 2010 [cited 2013 fev 15];15(2):278-85. Available from: http://dx.doi.org/10.5380/ce.v15i2.17862. 6. Vicente MG, Freitas DRC. Comportamento dos materiais médico-hospitalares no Brasil a partir dos dados de notificação em Tecnovigilância. Boletim Informativo de Tecnovigilância. [Internet]. Brasília; 2012 [cited 2014 Jul 28]. Available from: http://www.anvisa.gov.br/boletim_tecno/boletim_tecno_a gosto_2012/PDF/3BIT-4-2012-

Comportamento\%20Materiais\%20M\%C3\%A9dicohospitalares.pdf

7. Ministério da Saúde (BR). Agência Nacional de Vigilância Sanitária. Produtos para saúde. [Internet]. Brasília; 2013. [cited 2013 abr 17]. Available from:

http://portal.anvisa.gov.br/wps/content/Anvisa+Portal/Anvisa/Ini cio/Produtos+para+Saude

8. Ministério da Saúde (BR). Agência Nacional de Vigilância Sanitária. Manual de tecnovigilância: abordagens de vigilância sanitária de produtos para a saúde comercializada no Brasil [Internet]. Brasília; 2010. [cited 2013 abr 17]. Available from: http://portal.anvisa.gov.br/wps/wcm/connect/378e9d00474587a f9170d53fbc4c6735/manual_tecnovigilancia.pdf?MOD=AJPERES 9. Trindade E, Vicente MG, Santanna PC, Melchior SC, Pismel AMCL, Tinoco ATM, et al. Modos de falhas de artigos médicohospitalares: análise das queixas técnicas envolvendo equipos de infusão notificadas à ANVISA em 2007 e 2008. BIT: Boletim Informativo de Tecnovigilância [Internet]. 2010 [cited 2013 fev 19]. Available from:

http://www.anvisa.gov.br/boletim_tecno/boletim_tecno_dez_201 0/boletim_tecno.html

10. Trindade E, Vicente MG, Hofmeister MGSA, Cruz CJF, Hayashi ELO.; Matos MC, et al. Estudo multicêntrico de pré-qualificação: estudo de caso sobre equipos. BIT: Boletim Informativo de Tecnovigilância [Internet]. 2010 [cited 2013 fev 19]. Available from:

http://www.anvisa.gov.br/boletim_tecno/boletim_tecno_dez_201 0/boletim_tecno.html

11. Trindade E, Vicente MG, Santanna PC, Melchior SC, Pismel AMCL, Tinoco ATM, et al. A importância da tecnovigilância no processo regulatório de luvas no Brasil. BIT: Boletim Informativo de Tecnovigilância [Internet]. [cited 2013 abr 17];2011;(2). Available from:

http://www.anvisa.gov.br/boletim_tecno/boletim_tecno_Junho_2 011/PDF/A\%20import\%C3\%A2ncia\%20da\%20Tecnovigil\%C3\%A 2ncia\%20no\%20processo $\% 20$ regulat $\%$ C3\%B3rio $\% 20$ de $\% 20 l u v a$ s\%20no\%20Brasil.pdf

12. Ministério da Saúde (BR). Agência Nacional de Vigilância Sanitária. Manual de pré-qualificação de artigos médicohospitalares: estratégia de vigilância sanitária de prevenção. Brasília (DF); 2010. [citado 2013 nov 10]. Disponível em: http://portal.anvisa.gov.br/wps/wcm/connect/1776d7804745929 f9b00df3fbc4c6735/Capa+-+Manual+de+Pr\%C3\%A9+Qualifica\%C3\%A7\%C3\%A30.pdf?MOD=AJPERES 13. Zuliani, LL; Jericó, MC; Castro, LC; Soler, ZASG. Consumo e custo de recursos materiais em unidades pediátricas de terapia intensiva e semi-intensiva. Rev. bras. enferm. [Internet]. 2012 [cited 2014 Ago 10]; 65(6): 969-76. Available from: http://www.scielo.br/pdf/reben/v65n6/a13v65n6.pdf 14. Gil RB. O processo de notificação da queixa técnica de material de consumo de uso hospitalar no contexto do gerenciamento de recursos materiais em um hospital universitário público [dissertation]. Ribeirão Preto: Escola de Enfermagem de Ribeirão Preto, Universidade de São Paulo; 2011. [cited 2013 jul 03]. Available from: http://www.teses.usp.br/teses/disponiveis/22/22132/tde29082011-150211/

15. Ministério da Saúde (BR). Agência Nacional de Vigilância Sanitária. Participação dos profissionais de Saúde [Internet]. Brasília; 2013 [cited 2013 abr 22]. Available from:

http://www.anvisa.gov.br/servicosaude/hsentinela/participacao profissionais.htm

16. Dallora MELV, Forster AC. A importância da gestão de custos em hospitais de ensino - considerações teóricas. Medicina (Ribeirão Preto) [Internet] 2008 [cited 2014 jul 29]; 41 (2): 135-42. Available from:

http://revista.fmrp.usp.br/2008/VOL41N2/rev_a_importancia_ge stao_custos_hospitais_ensino.pdf

17. Garcia SD, Gil RB, Haddad MCL, Vannuchi MTO, Costa DB. O enfermeiro no gerenciamento de material médico-hospitalar: revisão integrativa. Online Braz ] Nurs [Internet]. 2013 [cited 2013 jul 10]; 12 (2):411-26. Available from:

http://www.objnursing.uff.br/index.php/nursing/issue/view/35/s howToc

18. Garcia, SD; Haddad, MCL; Dellaroza, MSG; Costa, DB Miranda, JM. Gestão de material médico-hospitalar e o processo de trabalho em um hospital público. Rev Bras Enferm [Internet] 2012 [cited 2014 Jul 28]; 65(2):339-46. Available from: http://www.scielo.br/scielo.php?pid=S0034-

71672012000200021\&script $=$ sci_arttext

19. Resolução da Diretoria Colegiada RDC N05, de 4 de fevereiro de 2011 (BR). Estabelece os requisitos mínimos de identidade e qualidade para os tipos de luvas, sob regime de vigilância sanitária. [cited 2013 nov 18]. Available from:

http://www.sbpc.org.br/upload/conteudo/320110215103504.pdf 20. Resolução da Diretoria Colegiada RDC N04, de 4 de fevereiro de 2011 (BR). Estabelece os requisitos mínimos de identidade e qualidade para os equipos de uso único de transfusão, de infusão gravitacional e de infusão para uso com bomba de infusão. [cited 2013 nov 18]. Available from:

http://www.sbpc.org.br/upload/conteudo/320110215103504.pdf 21. Resolução da Diretoria Colegiada RDC N03, de 4 de fevereiro de 2011 (BR). Estabelece os requisitos mínimos de identidade e qualidade para as seringas hipodérmicas estéreis de uso único. [cited 2013 nov 18]. Available from:

http://www.sbpc.org.br/upload/conteudo/320110215103504.pdf 22. Melleiro MM, Tronchin DMR. Percepção de acompanhantesusuários e enfermeiros sobre qualidade assistencial em Unidades Pediátricas. Acta Paul Enferm [Internet]. 2010 [cited 2013 jul 10]; 23(5):646-51. Available from: http://dx.doi.org/10.1590/S0103-21002010000500010. 23. Hausmann M, Peduzzi M. Articulação entre as dimensões gerencial e assistencial do processo de trabalho do enfermeiro. Texto Contexto Enferm [Internet]. 2009 [cited 2013 jul 11]; 18(2):258-65. Available from:

http://www.scielo.br/pdf/tce/v18n2/08.pdf 24. Castilho V, Gonçalves VLM. Gerenciamento de recursos materiais. In: Kurcgant $P$, organizadora. Gerenciamento em enfermagem. Rio de Janeiro: Guanabara Koogan; 2010. p. 15567.

25. Kuwabara CCT, Évora YDM, Oliveira MMB. Gerenciamento de risco em tecnovigilância: construção e validação de instrumento de avaliação de produto médico-hospitalar. Rev. Latino-Am. Enfermagem [Internet]. 2010 [cited 2013 Nov 04]; 18(5):943951. Availablefrom: http://dx.doi.org/10.1590/S010411692010000500015.

Artigo recebido em 02/12/2013.

Aprovado para publicação em 01/07/2014.

Artigo publicado em 31/03/2015. 